\title{
»Of all English words awe is, I think, the one that expresses the fundamental religious fee- ling most nearly»
}

\section{Zur Idee der »Gottesfurcht» in der modernen Religionsfor- schung}

Peter Schüz

Bereits unter gebildeten Schriftstellern und Philosophen der Antike galt es als ausgemacht, dass zwischen Angst und Religion ein besonderes Verhältnis besteht. Weit verbreitet war schon damals der Slogan "primus in orbe deos fecit timor..." - "Im Anfang schuf die Angst die Götter...«. ${ }^{1}$ Dahinter steht der für religiöse Menschen eher ernüchternde Gedanke, die Religionen der Welt seien ihrem Wesen nach nichts anderes als kulturell verankerte Projektionen zur Überwindung oder Bewältigung der Ängste vor Krankheit, Tod, Schicksal und Naturgewalt. Besonders im Zeitalter der Aufklärung fand diese antike Hypothese vom angstkompensatorischen Ursprung der Religion zahlreiche Varianten, die bis heute gerne als "leitmotivisches Motto" in mehr oder weniger religionskritischen Schriften zitiert werden. ${ }^{2}$ Und tatsächlich ist kaum zu leugnen, dass das alte "geflügelte Wort" des »timor fecit deos" einen Grundzug der Religionsgeschichte umreißt: ${ }^{3}$ Zweifellos ging es in den Religionen der Welt zu allen Zeiten auch maßgeblich um Trost und um die Verarbeitung von Ängsten. Für das Christentum ergeben sich bereits beim Blick in kirchliche Gesangbücher entsprechen-

1 Vgl. z.B. die vielzitierte Stelle bei Petronius, Fragment XXVIII, 1, in: Konrad Müller (Hg.), Petronii Arbitri Satyricon Reliquiae, 184 (= in der klassischen Ausgabe von Franz Bücheler: Fragm. XXVII, 1). Zur Wirkungsgeschichte vgl. Heinrich von Stietencron, Von der Heilsträchtigkeit der Angst. Religionsgeschichtliche Perspektiven, in: Ders. (Hg.), Angst und Religion, Düsseldorf 1991, 13-36 und Axel Michaels, Religionen und der neurobiologische Primat der Angst, in: Fritz Stolz (Hg.), Homo naturaliter religiosus. Gehört Religion notwendig zum Mensch-Sein?, Studia Religiosa Helvetica 97, Bern 1997, 91-136.

2 Zur kritischen Beurteilung des "leitmotivischen Mottos" vor dem Hintergrund der Geschichte der Religionskritik vgl. Winfried Schröder, Ursprünge des Atheismus. Untersuchungen zur Metaphysik- und Religionskritik des 17. und 18. Jahrhunderts Bd. 11, Stuttgart-Bad Cannstatt 1998, 153 u. 225.

3 Martin Persson Nilsson, Geschichte der griechischen Religion, Bd. 2, Die hellenistische und römische Zeit, München ${ }^{4} 1988,251$. 
de Beispiele für die auf Angstbewältigung zielende Hoffnung von den Paul-Gerhardt-Klassikern des 17. Jahrhunderts bis $\mathrm{zu}$ den Liebe-wie-Gras-und-Ufer-Schlagern der 1960er und 1970er Jahre. Bekanntlich bemerkte schon Luther ganz nüchtern: "So verhält es sich ganz natürlich: vom Unglück befallen, wenden sich die Seelen zur Religion «. ${ }^{4}$ Die vertrauensvolle Artikulation von Angst und ihre Wendung in hoffnungsvolles Vertrauen hat im Christentum ihren festen Ort in Seelsorge, Gottesdienst und gelebter Frömmigkeit. Religion deshalb aber allein auf ihre Funktion als psycho-soziales Angst-Therapeutikum zu reduzieren, wäre voreilig. Tiefere Einblicke in die Religions- und Frömmigkeitsgeschichte lassen erahnen, dass die Geschichte von Angst und Religion damit noch längst nicht auserzählt ist.

Besonders die moderne Bibelwissenschaft konnte zeigen, dass Momente der Angst in den Schriften des Alten und Neuen Testaments keineswegs nur als zu überwindendes Lebensproblem vorkommen - ganz im Gegenteil: An zentralen Stellen ist vielmehr von besonderen Momenten der Angst die Rede, die nicht gelindert oder überwunden, sondern geradezu in den Rang vollendeter Frömmigkeit erhoben werden. Auch stattliche Propheten erzittern an heiligen Orten, Momente der "Gottesfurcht" oder des »Zornes Gottes" markieren entscheidende Höhepunkte in den biblischen Überlieferungen. In zahlreichen Varianten hebräischer und griechischer Begriffe wird das Gottesverhältnis des Menschen in seinem Idealzustand als besonderer Moment frommer "Angst" geschildert. Die Furcht Gottes gilt als »der Weisheit Anfang « ${ }^{5}$ und begleitet die machtvolle Präsenz Gottes an heiligen Orten, in Träumen und Epiphanien. Nicht die Furcht vor Strafe oder Willkür, sondern das Gott-Sein Gottes selbst ist hier der Grund jenes heiligen Erschauderns. »Furcht und Zittern ${ }^{6}$ gilt schon bei Paulus als die eigentlich angemessene Gottesbegegnung und auch der Eindruck Jesu auf seine Anhänger und Zuhörer führte, wie die Evangelien beschreiben, oft genug dazu, dass jene »sich ängsteten " oder »entsetzten «. ${ }^{7}$ Die Kirchenväter haben sich mit den besonderen Angstmomenten in der Begegnung mit dem Heiligen eingehend befasst - man denke nur an Laktanz, Augustin und andere. Immer wieder geht es seither in den großen Werken der Theologiegeschichte bis zu Luther und darüber hinaus

\footnotetext{
4 Vgl. WA 25, 359,33-34: "Sic autem naturaliter fit: ingruente calamitate ad religionem convertuntur animi."

5 Ps $111 /$ Spr $1,7$.

6 Phil 2,12.

7 Vgl. Mk 4,41, Mk 10,32, Mt 17,6, Lk 2,9, Lk 5,26 u.v.m.
} 
um die Darstellung jener eigentümlichen "Gottesfurcht", die keine natürliche Ursache der Bedrohung, sondern die eigentümliche Heiligkeit und Unfassbarkeit Gottes zum Gegenstand hat.

In der modernen Religionsforschung hat man diese Tiefendimension religiöser Angstmomente in ihrer psychologischen Komplexität und in ihren religionsgeschichtlichen Darstellungsformen konzentriert in den Blick genommen und untersucht. Im Folgenden soll in aller Kürze an zwei prominente Stimmen erinnert werden, die vor etwa 100 Jahren das antike Motto, es habe die Angst die Götter hervorgebracht, in seiner reduktionistisch-religionskritischen Einseitigkeit zurückwiesen und das Zusammenspiel von Angst und Religion im Rahmen ihrer religionsgeschichtlich-religionspsychologischen Studien als einen Urmoment ganz eigener Art und Qualität deuteten: zunächst der britische Philosoph und Anthropologe Robert Ranulph Marett und sodann der protestantische Theologe Rudolf Otto.

\section{Der »bull-roarer« und andere Schauergeschich- ten: R. R. Maretts »Awe« Konzeption}

Um 1900 bildete Großbritannien eines der wichtigsten Zentren moderner Religionsforschung. Was man heute >Religionswissenschaft $\iota$, Ethnologie oder `Anthropologie nennen würde, verband sich auf der Insel mit Namen wie Edward Burnett Tylor oder James George Frazer, die im ausgehenden 19. Jahrhundert auf ein, wie man damals sagte, "naturalistisches" oder "evolutionistisches" Religionsverständnis hinauswollten: Man untersuchte Religionen vorwiegend als im Evolutionsprozess hervorgebrachte Kulturphänomene, die sich in ihrer sozialpsychologischen Funktion historisch und empirisch beschreiben lassen. Damit stand man ganz in der empiristisch-aufklärerischen Tradition von David Humes 1757 erschienener Natural History of Religion, in der ebenfalls primär die Angstbewältigung als Ursprung aller Religion angenommen wird. ${ }^{8}$ Umso erstaunter war die britische Fachwelt, als auf der Jahrestagung der British Anthropological Society von 1899 ein junger Philosoph aus

\footnotetext{
8 Hume schreibt hier ganz auf der Linie des antiken »timor fecit deos«-Leitmotivs: "The primary religion of mankind arises chiefly from an anxious fear of future events." (David Hume, The Natural History of Religion [1757], in: Thomas Hill Green/Thomas Hodge Grose (Hg.), David Hume, The Philosophical Works, Vol. 4: Essays. Moral, Political, and Literary, London 1882 (Reprint Aalen 1964), 307-363, hier 352f.).
} 
Oxford in seinem Vortrag plötzlich eine ganz andere Geschichte vom Wesen der Religion erzählte. Die These, die Robert Ranulph Marett ${ }^{9}$ (1866-1943) damals vortrug und die ihn in den folgenden Jahren berühmt machte, bezeichnete man schon bald als "PräAnimismus«: Anders als die von einer evolutionären Entwicklung der Religion aus Seelenvorstellungen (Animismus) ausgehenden Kollegen und Lehrer betonte Marett in seinem später erschienenen Buch The Threshold of Religion (1909) einen jeder religiösen Vorstellung vorausliegenden Urmoment der Religion, der weniger in Gedanken und Vorstellungen, sondern in Gefühlen und Intuitionen gründet, die durch den Intellekt letztlich nicht angemessen aufgelöst und beschrieben werden können. ${ }^{10}$ Für Marett ist »die ursprüngliche, wilde Religion weniger etwas Ausgedachtes, als vielmehr etwas Ausgetanztes. $\|^{11}$ Das »raw material of religion « hält er für einen dunklen »organischen Komplex von Denken, Emotion und Verhalten. ${ }^{12}$ Der hermeneutische Kniff, um jene Grundkonturen der Religion zu umschreiben, besteht für Marett nun darin, Begriffe zu wählen, die selber dunkel und aus der Welt "primitiver" Südsee-Religion entlehnt sind: Der Begriff "tabu« steht für das sich Entziehende, Unantastbare, Abdrängende, Negative an religiösen Orten, Handlungen, Gegenständen und Personen, der Begriff "mana" hingegen für die positiven Momente, für Macht, Affektives, Anziehendes, Erhebendes. Hinsichtlich der Erforschung des Ursprungs der Religion ist Marett demnach weitaus pessimistischer und vorsichtiger als die Generation seiner Lehrer: Er hält das »erste Kapitel der Religionsgeschichte» für "undechiffrierbar « ${ }^{13}$ Mag sfertige Religion auch sozial nützliche und angstbewältigende Nebenwirkungen haben: Ihr Ursprung ist alles dies nicht, sondern gleicht einer amorphen, dynamischen Intuition, die vor jeder Reflexion und Vorstellung liegt und keine snützlichen` Zwecke verfolgt. Anders als seine Vorgänger bis hin zu Hume übersetzt Marett daher das antike »deos fecit timor«-Motto nun auch nicht reduktionistisch-religionskritisch mit "Furcht» oder "Angst« (fear/anxiety), sondern wählt den sehr viel weiteren und psychologisch vageren Begriff »awe» (Scheu, Schauder, Ehrfurcht):

\footnotetext{
9 Vgl. insbes. Maretts Autobiographie: Robert Ranulph Marett, A Jerseyman at Oxford, Oxford 1941.

${ }^{10}$ Vgl. den wichtigen, alle Programmaufsätze bündelnden Sammelband: Robert Ranulph Marett, The Threshold of Religion, $\left({ }^{1} 1909\right)$ second Edition, revised and enlarged, New York 1914.

${ }^{11}$ Marett, The Threshold of Religion, XXXI (Übers. v. P. Schüz).

12 Ebd., X (Übers. v. P. Schüz).

${ }^{13}$ Ebd.,VIII (Übers. v. P. Schüz).
} 
"Of all English words awe is, I think, the one that expresses the fundamental religious feeling most nearly. Awe is not the same thing as spure funk we admit wonder, admiration, interest, respect, even love perhaps, to be, no less than fear, essential constituents of this elemental mood. ${ }^{14}$

Das antike Leitmotiv »Primus in orbe deos fecit timor» ist demnach "nur insofern wahr, als wir Verwunderung, Staunen, Interesse, Respekt, vielleicht sogar Liebe in gleichem Maße wie Furcht als essentielle Bestandteile dieser Elementarstimmung hinzunehmen. $«{ }^{15}$ Dabei geht es Marett keineswegs nur um semantische Spitzfindigkeiten, sondern um ein Kategorienproblem. In seinen zahlreichen Studien zu religiösen Phänomenen in unterschiedlichen Kulturkontexten (insbes. in Afrika und Australien) versucht Marett zu zeigen, dass die Welt der Religionen keineswegs aus sozialen oder spekulativen Phantasien zur Lebensbewältigung und Angstreduktion hervorgeht, sondern weit darunter vor jeder bewussten Darstellung und Ausdrucksform als dunkle Intuition zwischen "tabu« und "mana" lebendig ist. ${ }^{16}$ Das »basic feeling of awe« ist für Marett exklusiv, "specifically religious both in its essence and in its fruits «. ${ }^{17} \mathrm{Hierzu}$ erkundete Marett Rituale unterschiedlicher Religionskulturen wie z.B. den Gebrauch des australischen »bull-roarers«, eines archaischen Schwirrholz-Instruments der Aborigines zur Erzeugung besonderer Laute im kultischen Kontext, das seiner Auffassung nach eben jene dynamisch-dunklen Schauergefühle von "awe" anregen und spirituell einbinden soll. ${ }^{18}$ Mit seinen Studien zu jener ganz besonderen Angststimmung als »Schwelle zur Religion« ist Marett zu einem Klassiker der modernen religionsphänomenologischen Forschung geworden, der, auch wenn seine Argumentation heute meist als "überholt" gilt, in kaum einem Kompendium zur Geschichte der Religionswissenschaft fehlt. ${ }^{19}$

\footnotetext{
${ }^{14}$ Ebd., 13 (Hervorhebung im Original).

15 Ebd., 13 (Übers. v. P. Schüz).

${ }^{16}$ Vgl. hierzu insbes. die Oxforder Marett Lecture 2014 (Exeter College): Birgit Meyer, How to capture the swow . R.R. Marett's notion of awe and the study of religion, in: JRAI 22 (2016), 7-26.

${ }_{17}$ Marett, The Threshold of Religion, 14f.

${ }_{18}$ Vgl. den erstmals 1910 erschienen Aufsatz Supreme Beings and the Bull-Roarer, abgedruckt in: Marett, The Threshold of Religion, 145-168.

${ }^{19}$ Siehe neben Martin Riesebrodt, Robert Ranulph Marett (1866-1943), in: Axel Michaels (Hg.), Klassiker der Religionswissenschaft. Von Friedrich Schleiermacher bis Mircea Eliade, München 1997, 171-184 besonders die luzide Darstellung von
} 


\section{R. Ottos »numinose Scheu« als artbesondere Deutungs- und Bewertungskategorie}

Im Herbst des Jahres 1916 - mitten im Ersten Weltkrieg - fiel Maretts Threshold of Religion erstmals in die Hände des im Jahr zuvor als Professor nach Breslau berufenen Theologen Rudolf Otto (1869-1937). Seinen wissenschaftlichen Schwerpunkten der vorangehenden Jahre in Göttingen folgend, legte Otto in der Vorbereitung auf das Wintersemester 1916/1917 einen deutlich religionsgeschichtlichreligionspsychologisch gefärbten Fokus für seine Lehrveranstaltungen. Was Otto nun bei Marett las, ließ ihn sofort eine gewisse Geistesverwandtschaft zu jenem Werk entdecken, dessen Manuskript er kurz zuvor im weitgehend unbekannten BreslauerVerlag Trewendt \& Granier eingereicht hatte und von dem damals noch niemand ahnte, dass daraus einmal einer der großen theologischen Klassiker des 20. Jahrhunderts werden würde. Das Buch Das Heilige wurde schon bald in viele Sprachen übersetzt und machte Rudolf Otto weit über Fach- und Landesgrenzen hinaus bekannt. ${ }^{20}$ Am 5. Dezember 1916 schrieb er an Wilhelm Bousset, seinen nun in Gießen lehrenden Freund aus Göttinger Tagen:

"Haben Sie Marett, threshold of Religion, gelesen? Mir kam es erst zur Hand, als ich mit dem Heiligen fertig war. Es ist prachtvoll, wie jetzt auch die Naturalisten, wenn sie nur die Augen aufmachen, herumkommen. Das Buch ist wirklich ein Denkstein in der Erforschung der Ursprünge der Religion. $\mathbb{1}^{21}$

Besonders ein Gedanke wird es gewesen sein, der Otto an Maretts „Präanimismus« besonders einleuchtete: Die Idee, dass der dunkle Urgrund aller Religion im Gefühl einer besonderen, von gewöhnlicher Angst zu unterscheidenden "Scheu" (bei Marett: "awe«) zu suchen sei, in der die Religion, wie Otto immer wieder betont, "mit sich selber anfängt" und damit ihren Grund voraussetzungslos in sich selber trägt. ${ }^{22}$ Was für Otto im Zuge "moderner Erforschung

Hans G. Kippenberg, Die Entdeckung der Religionsgeschichte. Religionswissenschaft und Moderne, München 1997, 179-193.

${ }^{20}$ Die Erstausgabe Rudolf Otto, Das Heilige. Über das Irrationale in der Idee des Göttlichen und sein Verhältnis zum Rationalen, Breslau 1917 erlebte zahlreiche Auflagen, Überarbeitungen und Verlagswechsel bis zur Ausgabe letzter Hand bei C.H. Beck, München ${ }^{23-25} 1936$.

${ }^{21}$ Vgl. den maschinengeschriebenen Brief von Rudolf Otto an Wilhelm Bousset vom 5.12.1916, UB Göttingen, Nachlass Bousset 90/2.

${ }^{22}$ Vgl. z.B. Rudolf Otto, Der sensus numinis als geschichtlicher Ursprung der Religion, in: Ders., Das Gefühl des Überweltlichen (sensus numinis), München 1932, 
der >Primitiv`-formen" in der Religionsgeschichte "immer klarer ins Licht" kommt und "seit Maretts vortrefflichem Buche: >The threshold of religion nicht mehr übersehen werden" darf, ist jene Intuition, die in seinem Hauptwerk zu der berühmten Formel des "mysterium tremendum et fascinans" führte in dem Gedanken, dass auch und gerade in vermeintlich "primitiven" Religionsstrukturen "echtes religiöses Fühlen mit wahrer `Andacht` und Hingabe vorhanden sein" kann. ${ }^{23}$ Gemeint ist eine ganz eigene Erlebniskategorie der "numinosen Scheu" im Sinne einer Erkenntnis a priori, die damit epistemologisch vor jeder Erfahrung gewöhnlicher, auf Sinnesreizen oder Gedanken der Bedrohung beruhender Angst oder Furcht liegen muss. ${ }^{24}$ Jener Sphäre des Numinosen galt das Hauptanliegen von Ottos Hauptwerk über das "Irrationale in der Idee des Göttlichen und sein Verhältnis zum Rationalen" - und schlug sich, wie bei Marret, der laut Otto »der Sache auf Haaresbreite nahe $\aleph^{25}$ kam, in einer charakteristischen Neudeutung des alten "deos fecit timor«-Mottos nieder:

"- 'Scheu ist dieses Gefühl auf niedrigster Stufe. Aber schon als erstes elementares Grauen vor dem `Unheimlichen ist es schon ein Scheuen völlig eigener Art, typisch verschieden von 'Furcht in gewöhnlichem Sinne: eher im Anfange ein ๖blindes Entsetzen`, nämlich ein solches, bei dem ganz dunkel bleibt, wovor und was man eigentlich scheut, aber nicht Furcht im gewöhnlichen Sinne.Von allem Anfang scheut der Mensch - und zwar in ganz unsagbarer Weise - ein Gewisses, zunächst ganz Unausgesprochenes mehr als Tod und Untergang, mehr als alles, 'was nur den Leib zu verderben vermag $₫$. Nicht stimor fecit deos`sondern

11-57, hier 53: „So fängt Religion zwar nicht als fertige Religion wohl aber mit sich selbst an, sofern sie, als sensus numinis von Anbeginn Erlebnis des Mysteriösen und Zug und Trieb zum Mysterium ist, ein Erleben, das aus den Tiefen des Gefuhlslebens selber, auf Reize und Anlässe von außen hin, als das `Gefühl des Ganz andern durchbricht."

${ }^{23} \mathrm{Vgl}$. Otto, Der sensus numinis, 45.

${ }^{24}$ Ausführlich zur Idee der »numinosen Scheu« bei Otto und ihrem Verhältnis zu Angst/Furcht siehe auch Peter Schüz, Mysterium tremendum. Zum Verhältnis von Angst und Religion nach Rudolf Otto, BHTh 178, Tübingen 2016, sowie Ders., Heilige Scheu als religiöses Urphänomen. Das Dämonische und das Numinose, in: Christian Danz/Werner Schüßler (Hg.), Das Dämonische. Kontextuelle Studien zu einer Schlüsselkategorie Paul Tillichs, Bd. 15, Berlin/Boston 2018, 41-68 und Ders., Numinose "Scheu« als "artlich andere Zuständlichkeit«. Rudolf Otto und der moderne Angstbegriff, in: Jörg Lauster u.a. (Hg.), Rudolf Otto. Theologie - Religionsphilosophie - Religionsgeschichte, Berlin/Boston 2014, 127-142.

25 Otto, Das Heilige, 17. 
jene S Scheu wurde - auf wunderliche und uns vielfach nur mühsam verständliche Weise aufgeregt - zum Antriebe, den sie begleitenden dunklen und nur gefühlten Vorstellungs-inhalt fantasiemäßig-analogisch zu vergegenständlichen in Vorstellungen und Symbolen, die eben deswegen auf allen Stufen schwebenden, fließenden, begrifflicher Festlegung widerstehenden und zugleich aus sich heraus vorwärts drängenden Charakters sind. ${ }^{26}$

Otto geht es hier eindeutig nicht um alltägliche, existentielle Angst, die Projektionen beschwichtigender Gottesbilder heraufbeschwört, sondern um eine intuitive, vorreflexive "Deutungs- und Bewertungs-kategorie " ganz eigener Art. ${ }^{27}$ Als christlicher Theologe folgt Otto dabei also - anders als Marett - nicht allein der Phänomenbeschreibung religiöser Ausdrucks- und Darstellungsformen, sondern, im Rückgriff auf Kant, Fries, Schleiermacher und de Wette, auch der Annäherung an den tieferen religiösen Wert der Sache, an das eigentliche Wesen der Frömmigkeit. Otto will sagen können, was Gegenstand und Quelle dieser "Scheu« ist, die er besonders im Alten Testament im heiligen Erschaudern der Propheten entdeckte und die ihm dann in allen Epochen der Christentumsgeschichte bei Gestalten wie Chrysostomos und Augustinus, in Traditionen der Mystik, ganz besonders bei Luther und schließlich auch außerhalb des Christentums und auf seinen Reisen um die halbe Welt begegnete. Als Wurzel der ganz artbesonderen "numinosen Scheu « machte Otto schließlich etwas aus, was er nicht anders als eine Begegnung mit dem Mysterium des Heiligen umschreiben konnte. Der Grund des heiligen Erschauerns und der frommen Scheu in der Religion ist seiner Auffassung nach kein Objekt oder Reiz, keine natürliche oder übernatürliche Ursache im eigentlichen Sinne, sondern die Intuition für das, was er das "Ganz andere " nannte, das religiöse Mysterium schlechthin. ${ }^{28}$ Letztlich ist es damit eine besondere Art der Selbsterkenntnis, die sich in jenen Momenten ereignet, die man traditionell als Gottesfurcht bezeichnet: Otto spricht vom »Kreaturgefühl«, in dem sich der Mensch verloren und nichtig erlebt im Gegenüber einer intuitiv erahnten und erlebten Macht des »Ganz anderen " als dem entscheidenden Urdatum der Religion und - in Begriffen wie Sünde, Buße und Sühne - als Dreh- und Angelpunkt des christlichen Glaubens. ${ }^{29}$

\footnotetext{
${ }^{26}$ Otto, Der sensus numinis, $52 \mathrm{f}$.

${ }^{27}$ Otto, Das Heilige, 7.

${ }^{28} \mathrm{Zu}$ diesem Grundbegriff Ottos vgl. insbes. das Kapitel über das "Ganz andere« in ebd., 28-37.

${ }^{29}$ Zum »Kreaturgefühl« vgl. insbes. ebd., 8-12.
} 


\section{Der Weisheit Anfang. Die Idee der Gottesfurcht als dunkles Erbe des Christentums}

Es ist vermutlich kaum ein Zufall, dass die geschilderte religionspsychologische (Wieder-) Entdeckung der Gottesfurcht bezeichnenderweise genau in jene Zeitepoche fällt, in der es zu tiefgreifenden Umbrüchen in der Wahrnehmung traditioneller Begriffe und Lehren des christlichen Glaubens kam. Vor dem Hintergrund der vielbeschworenen Entzauberungsprozesse der Moderne erscheinen die Erkundungen von Marett und Otto wie Rückbesinnungen auf ein in Vergessenheit geratendes Erbe religiösen Lebens, dem man, wenn ich richtig sehe, auch heute zuweilen etwas ratlos gegenübersteht. Die uralte Rede von heiliger Scheu, von Sünde und Sühne, vom Zorn Gottes und dem kaum zu überblickenden Spektrum der sich daraus ergebenden frommen Ausdrucks- und Darstellungsformen in der Religionsgeschichte überfordert zuweilen die spirituellen Erwartungshaltungen der Gegenwart. Das Erbe der Gottesfurcht wird häufig auch als anstößige Zumutung empfunden, die überdies in der Botschaft von Kreuz und Passion bis an den Kern der christlichen Botschaft heranreicht, ja vielleicht sogar, wenn man Paulus beim Wort nimmt, dessen Pointe markiert. ${ }^{30}$ Vor diesem Hintergrund kommt der von Marett und Otto verfolgten Spur zu den dunklen Dimensionen und schauervollen Untiefen der Religion womöglich eine hermeneutische Schlüsselfunktion zu, die darin besteht, auf die eigentlichen und seit Jahrtausenden lebendigen Triebkräfte religiöser Darstellung und Frömmigkeit hingewiesen zu haben, aus denen die zuweilen archaische und dunkle Welt spiritueller Ausdrucksgestalten überhaupt erst hervorgegangen ist. Entscheidend ist dabei an ihren Beobachtungen die scharfe Unterscheidung des religiösen Erbes frommer Gottesfurcht von gewöhnlicher Angst vor Bedrohung und Verderben. Zum Verstehen der Religion in Geschichte und Gegenwart gehört demnach, so ließe sich vielleicht resümieren, unweigerlich auch das Aneignen ihrer ganz eigenen, innerlichen Momente schauervollen Furchterlebens, die in alten heiligen Schriften, Orten und Bildern ebenso bewahrt sind, wie in der je eigenen, individuellen und innerlichen Lebensgeschichte religiöser Menschen aller Zeiten und Generationen. Ob sich am Ende durch

\footnotetext{
${ }^{30}$ Vgl. das berühmte Pauluswort von der Anstößigkeit ( $\left.\sigma \kappa \alpha ́ v \delta \alpha \lambda \circ v\right)$ und der Torheit

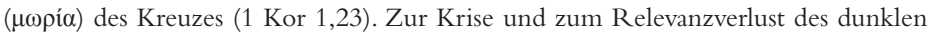
Erbes christlich-dogmatischer Klassiker vgl. u.a. die Debatte um das Buch von KlausPeter Jörns, Notwendige Abschiede. Auf dem Weg zu einem glaubwürdigen Christentum, Gütersloh ${ }^{2} 2005$.
} 
ein Abschreiten jener innerlichen Grenzregionen des Erlebens von "awe" und "numinoser Scheu " auch eine Wiedergewinnung der tieferen Lebendigkeit traditioneller dogmatischer und biblischer Rede und Darstellung gewinnen lässt, muss offen bleiben bzw. wäre erst noch auszuprobieren, - denn mag die Furcht Gottes auch »der Weisheit Anfang« sein (Ps 111), so ist sie letztlich vor allem dies: ein unergründliches, frommes Geheimnis. ${ }^{31}$

- Dr. Peter Schüz ist Akademischer Rat für Systematische Theologie an der Evangelisch-Theologischen Fakultät der Ludwig-Maximilians-Universität München. $\mathrm{Zu}$ seinen Arbeitsgebieten gehören neben Dogmatik und Religionsphilosophie Schwerpunkte zur Religionsästhetik, Frömmigkeitstheorie, Ökumene und interdisziplinären Religionsforschung.schuez@evtheol.uni-muenchen.de

${ }^{31}$ Zum weiterfuihrenden Hintergrund vgl. den Beitrag Peter Schüz, Das Kreuz als „Bild der Unversöhnlichkeit«. Überlegungen zum unmittelbaren Eindruck des Todes Jesu und seiner Bedeutung für die Christologie, in: Christian Danz/Michael Hackl (Hg.), Transformation der Christologie. Herausforderungen, Krisen und Umformungen, Bd. 17, Göttingen 2019, 61-88. 